\title{
Температурная зависимость проводимости нитевидных кристаллов теллура
}

\author{
() М.P. Рабаданов ${ }^{1}$, А.А. Степуренко ${ }^{2}$, А.Э. Гумметов ${ }^{2}$, А.М. Исмаилов ${ }^{1,9}$ \\ ${ }^{1}$ Дагестанский государственный университет, \\ 367000 Махачкала, Россия \\ ${ }^{2}$ Институт фризики, Дагестанский фредеральный исследовательский центр Российской академии наук, \\ 367015 Махачкала, Россия \\ I E-mail: egdada@mail.ru
}

Поступила в Редакцию 12 февраля 2021 г.

В окончательной редакции 22 февраля 2021 г.

Принята к публикации 22 февраля 2021 г.

В интервале температур 77-273 K проведен сравнительный анализ электропроводности нитевидного кристалла, эпитаксиальной пленки и монокристалла теллура. Электропроводность пленки и монокристалла растет монотонно в диапазоне $77-200 \mathrm{~K}$, после чего начинается резкий рост, соответствующий термическому возбуждению собственных носителей заряда. Электропроводность нитевидных кристаллов теллура уменьшается в интервале температур $77-236 \mathrm{~K}$, затем следует плавный ее рост. Предполагается, что в случае нитевидных кристаллов теллура имеет место классический размерный эффект: спад электропроводности обусловлен диффузным рассеянием носителей боковой поверхностью образца и его усилением с ростом температуры. Развитая поверхность боковых граней нитевидного кристалла подтверждается исследованием морфологии на растровом электронном микроскопе.

Ключевые слова: теллур, нитевидный кристалл, удельная проводимость, морфология поверхности, классический размерный эффект.

DOI: $10.21883 /$ FTP.2021.06.50913.9631

\section{1. Введение}

Теллур - один из элементарных полупроводников $p$-типа проводимости с узкой запрещенной зоной $\left(E_{g} \approx 0.34\right.$ эВ) и сильно анизотропной кристаллической структурой, которая и обусловливает уникальный набор его физико-химических свойств: пьезоэлектрических, фотоэлектрических, нелинейных оптических, термоэлектрических, каталитических и др. [1]. Низкая температура плавления Те $\left(452^{\circ} \mathrm{C}\right)$ позволила легко получать монокристаллы расплавными методами [2,3]. Метод термического вакуумного напыления был первым методом получения тонких пленок теллура и используется по сей день $[4,5]$. Ранние исследования монокристаллов и тонких пленок Те позволили определить основные фундаментальные свойства кристаллического теллура [6,7]. При этом без ответа остались некоторые экспериментальные факты, не нашедшие надежного объяснения до сих пор (отсутствие вымораживания свободных носителей заряда в кристаллах Те при низких температурах, вплоть до $0.05 \mathrm{~K}$; исключительно дырочный тип проводимости; аномальная зависимость сопротивления от магнитного поля при низких температурах; аномальные оптические свойства). Данные закономерности были установлены на образцах теллура низкого кристаллического качества, полученных расплавными методами, включая эксперименты по кристаллизации Те в условиях невесомости [3]. Основным препятствием на пути получения пленок Те высокого кристаллического качества термовакуумным методом остается тот факт, что паровая фаза теллура почти целиком состоит не из атомов Те, а из двухатомных молекул $\mathrm{Te}_{2}$ [8]. Низкая подвижность $\mathrm{Te}_{2}$ на ростовой поверхности при температурах подложки $<452^{\circ} \mathrm{C}$ приводит к осаждению пленок теллура низкого кристаллического качества.

Новый этап в исследовании кристаллического теллура начался с 2000 г., но уже в форме низкоразмерных структур (нитевидные кристаллы, нанотрубки, наноленты, квантовые точки) [9-11]. Большинство проведенных работ связано с исследованием механизмов зарождения и роста низкоразмерных структур Те, что имеет большое значение как для самой физики роста кристаллов, так и для практики. Предложенные методы выращивания низкоразмерных структур Те можно объединить в две большие группы: жидкофазные методы синтеза; методы осаждения из газовой фазы. На сегодняшний день при исследовании структур теллура внимание исследователей сосредоточено на вопросах их практического применения. В публикациях в основном описаны попытки создания лабораторных образцов приборных структур с использованием как массива нитевидных кристаллов (НК), так и отдельного НК Те (газовые сенсоры, полевые транзисторы, термоэлектрические, пьезоэлектрические устройства и др.) [9].

Начиная с 2017 г. изучается двумерный теллур (теллурен) - новейший представитель семейства двумерных материалов [12,13]. Уже показаны преимущества приборных структур, изготовленных на основе теллурена [13-15]. 
Ранее мы сообщали о получении эпитаксиальных пленок теллура высокого кристаллического качества методом термохимической активации в среде водорода [16]. Дальнейшая отработка технологических режимов позволила получить нитевидные кристаллы и микропластинки теллура. С использованием просвечивающей электронной микроскопии высокого разрешения многими исследователями установлена практически бездефектная кристаллическая структура НК (например, для НК Те в $[17,18])$. Считается, что дефекты в процессе роста НК вытесняются из объема на поверхность. Поэтому следует ожидать различия в структурно-чувствительных свойствах НК и классических объектов (монокристалл, пленка). К структурно-чувствительным свойствам относится проводимость при низких температурах. В связи с этим цель данной работы заключалась в исследовании температурной зависимости проводимости НК Те и ее сопоставление с таковой для пленки и монокристалла Те.

\section{2. Методика эксперимента}

Эпитаксиальные пленки и НК Те были получены методом термовакуумного испарения теллура в среде водорода [16]. Контролируемыми технологическими параметрами процесса кристаллизации являются: температуры зоны тигля $T_{2}$ и подложки $T_{1}$, величина перепада температуры между зонами $\Delta T=T_{2}-T_{1}$, давление водорода $P_{\mathrm{H}_{2}}$ в системе. В работе использовался исходный теллур высокой чистоты марки 6N (5N Plus, Канада). Для исследования были использованы три образца.

Образец 1 - НК Те шестигранной формы (площадь сечения 70 мкм $^{2}$, длина $2.4 \mathrm{Mм}$ ), выбранный из массива НК Те (рис. 1), полученных при следующих условиях: $T_{2}=600^{\circ} \mathrm{C}, T_{1}=430^{\circ} \mathrm{C}, \Delta T=170^{\circ} \mathrm{C}, P_{\mathrm{H} 2}=0.8$ атм. В качестве подложки использовалась микросетка (материал - нихром, диаметр нити 25 мкм, размер ячейки $25 \times 25$ мкм). Откалывание НК Те от подложки осуществляли в изопропиловом спирте при помощи ультразвука. Затем отобранный одиночный НК Те переносили на подложку из поликора с предварительно нанесенными методом магнетронного распыления контактными площадками из серебра $3 \times 8$ мм на расстоянии 2.4 мм друг от друга. НК Те и подведенная к его концам проволока из меди (100 мкм) закреплялись на контактной площадке с помощью припоя из чистого индия.

Образец 2 - эпитаксиальная пленка Те (отделенная от подложки) толщиной 13 мкм (рис. 2), полученная на свежем сколе слюды (мусковит) при следующих условиях: $T_{2}=600^{\circ} \mathrm{C}, T_{1}=430^{\circ} \mathrm{C}, \Delta T=170^{\circ} \mathrm{C}, P_{\mathrm{H} 2}=1.2$ атм.

Образец 3 - монокристалл теллура в форме параллелепипеда $(1 \times 1.5 \times 4.7$ мм $)$, вырезанный из бульбы, выращенной методом Чохральского. Электрические контакты к пленке и монокристаллу Те изготавливали также с помощью индия.

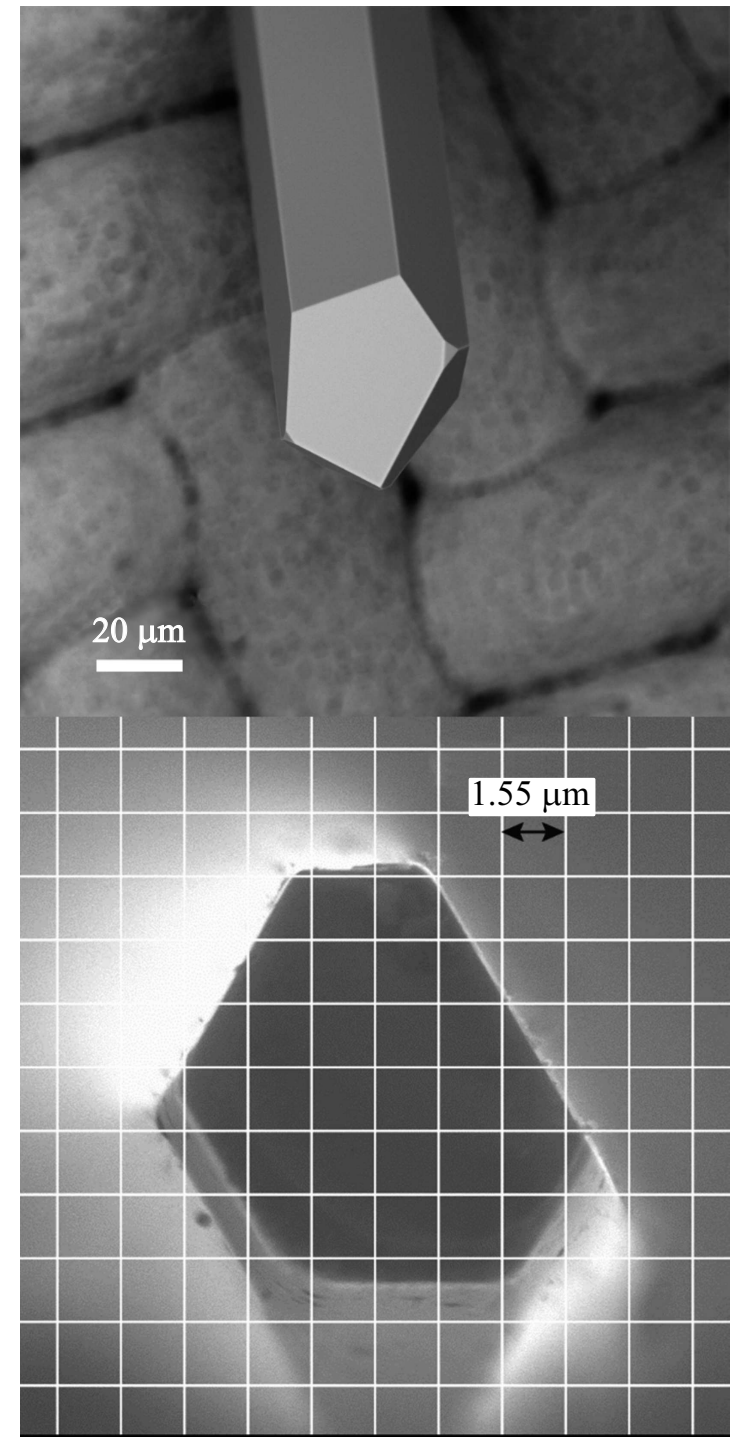

Рис. 1. Изображение в растровом электронном микроскопе типичного одиночного НК Те и поперечное сечение одиночного НК Те, отобранного для измерений.

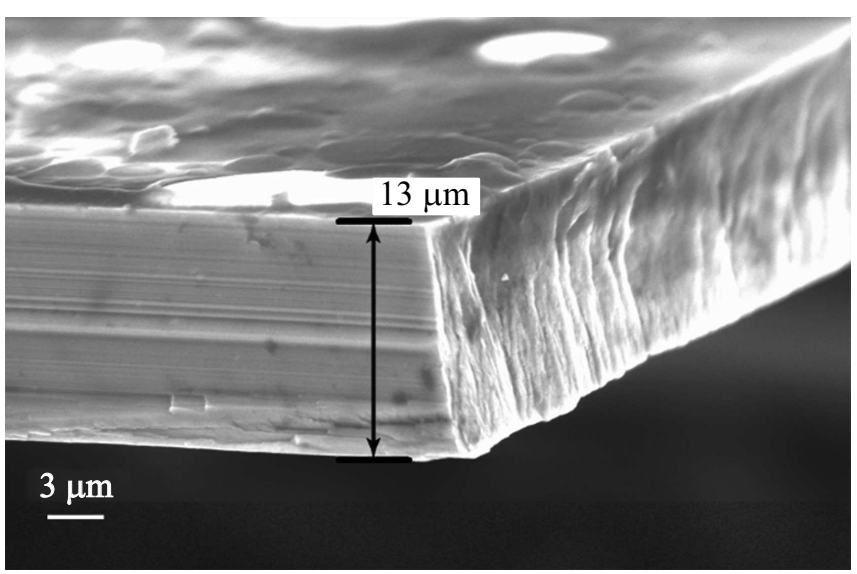

Рис. 2. Изображение в растровом электронном микроскопе эпитаксиальной пленки Те, отделенной от подложки из слюды (мусковит). 
Морфологическое исследование пленок и НК Те, а также измерение геометрических размеров образцов (рис. 1, 2) проводили на сканирующем электронном микроскопе SEM Leo-1450 (Zeiss, Германия) в режиме вторичных электронов. Рентгеновские исследования выполнялись на дифрактометре Empyrean Series 2 (PANalytical, Нидерланды) с использованием $\mathrm{Cu} K_{\alpha}$-излучения (длина волны $\lambda=0.15406$ нм). Спектры снимались в диапазоне углов $2 \theta=10-90^{\circ}$ в режиме непрерывного сканирования с шагом $0.01^{\circ}$ и со скоростью $0.5^{\circ} /$ мин.

Для измерения температурной зависимости проводимости образцы помещали в сосуд Дьюара с жидким азотом $(T=77 \mathrm{~K})$. Далее образцы вытягивали из жидкого азота и перемещали в парах азота в направлении градиента температуры $77<T<273 \mathrm{~K}$. Скорость перемещения образцов подбиралась так, чтобы температура образцов в данной точке в парах азота в момент измерения оставалась неизменной.

Температурные зависимости проводимости образцов были получены с использованием одиночных импульсов напряжения, приложенных к образцам, длительностью 10 мкс. Применение импульсной методики было обусловлено задачей свести к минимуму нагрев образца. Удельную проводимость рассчитывали по измеренному сопротивлению с учетом размеров образцов.

\section{3. Экспериментальные результаты и их обсуждение}

Дифрактограммы образцов теллура, использованных для исследования температурных зависимостей проводимости, представлены на рис. 3. Идентификация дифракционных рефлексов образцов проводилась на основе дифрактограммы исходного порошка теллура. Все дифракционные пики находятся в хорошем соответствии со стандартными данными для теллура (PDF-00-036-1452).

Из рис. 3 видно, что для всех трех образцов характерны узкие интенсивные рефлексы (100), (200), (300) при углах 23.2, 47.1, $73.7^{\circ}$ соответственно. Данные „отражения“ соответствуют тригональной структуpe теллура (пространственная группа $\left.\mathrm{D}^{4}{ }_{3}{ }^{(6)}\right)$. Их также можно рассматривать как пики 1-, 2- и 3-го порядков, соответствующие отражению от плоскости (100). Для эпитаксиальной пленки (образец 2) данная плоскость параллельна плоскости подложки, т.е. ось с кристаллического теллура лежит в плоскости подложки.

Для рентгеновских дифракционных исследований НК Те был подготовлен образец с взаимно параллельным расположением нитевидных кристаллов на стеклянной подложке. Для этого были отобраны НК Те диаметром 10-80 мкм, их средняя длина составляла 8 мм. Для их надежного закрепления на подложке был использован спиртоканифольный флюс. В дифрактометре образец был расположен так, чтобы пучок рентгеновского излучения был направлен вдоль НК, при этом вращение образца не проводилось (см. вставку на

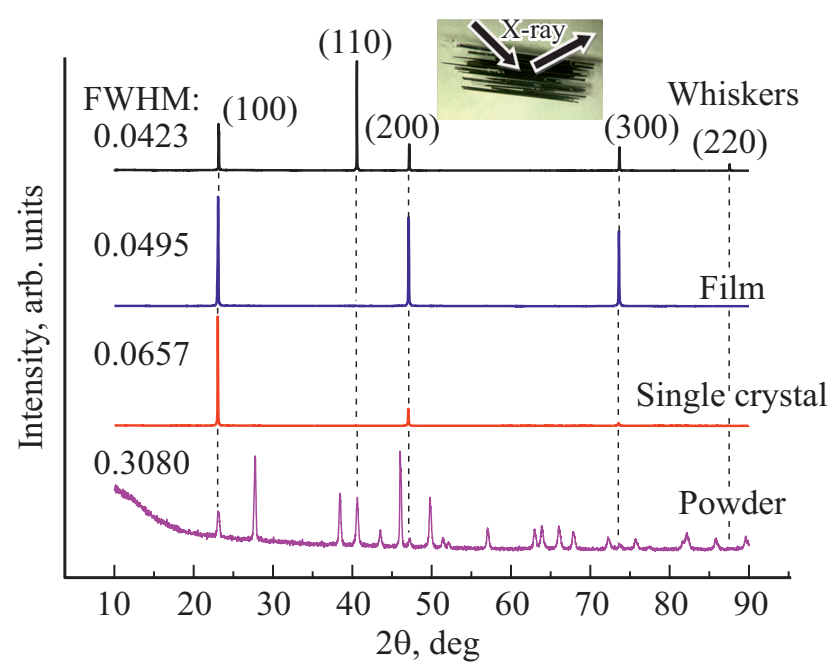

Рис. 3. Дифрактограммы образцов теллура.

рис. 3). Известно, что НК Те растут вдоль направления кристаллографической оси с. Поскольку для НК Те, лежащих параллельно поверхности подложки, поворот вокруг оси с - произвольный, то в „отражающем“ положении оказывается также и плоскость (110), которой соответствуют рефлексы 1- и 2-го порядков (110), (220) при углах $40.6^{\circ}, 87.6^{\circ}$ соответственно.

На основе анализа по сопоставлению отношений интенсивностей пиков (100), (200), (300) для одного образца и их полуширины (FWHM) для разных образцов можно сделать вывод о том, что по кристаллическому качеству НК Те превосходят эпитаксиальную пленку и монокристалл.

На рис. 4 представлены температурные зависимости проводимости $\sigma(T)$ НК, пленки и монокристалла теллура в интервале температур $T=77-273 \mathrm{~K}$. Видно, как электропроводность монокристалла постепенно растет до $\sim 200 \mathrm{~K}$, после чего начинается более крутой рост, соответствующий термическому возбуждению собственных носителей заряда. Температурная зависимость проводимости пленки, за исключением небольшой особенности в области $100-200 \mathrm{~K}$, подобна аналогичной зависимости для монокристалла. Однако температурная зависимость проводимости НК Те коренным образом отличается от кривых для пленки и монокристалла. С ростом температуры от 77 до $236 \mathrm{~K}$ наблюдается спад электропроводности НК Те, после чего наблюдается плавный рост до температуры $273 \mathrm{~K}$ (рис. 4, кривая 1).

Выражение для электропроводности твердых тел имеет вид $\sigma=e n \mu$, где $n-$ концентрация носителей заряда в образце, $\mu-$ их подвижность. Примем, что для нелегированного теллура, как и для всякого полупроводника, имеет место зависимость $n(T)=C e^{-E_{g} / 2 k T}$.

В работе [19] сообщается, что для пленок теллура в интервале от 85 до $250 \mathrm{~K}$ температурная зависимость подвижности имеет вид $\mu \propto T^{3 / 2}$, а концентрация носителей остается постоянной. Такая же зависимость подвиж- 


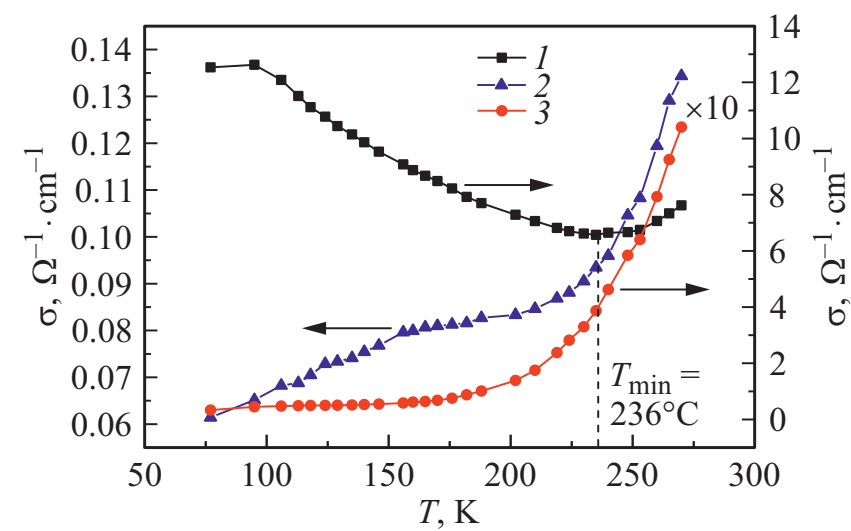

Рис. 4. Температурная зависимость проводимости образцов теллура: 1 - НК, 2 - эпитаксиальная пленка, 3 - монокристалл.

ности от температуры наблюдается и в монокристаллах теллура $[20,21]$. Поэтому имеются основания полагать, что и в нашем случае (для пленки и монокристалла) имеет место зависимость $\mu(T)=B T^{3 / 2}$. Тогда можно записать

$$
\begin{aligned}
\frac{d \sigma}{d T}= & e\left[\left(C B E_{g} / 2 k t^{2}\right) \cdot e^{-E_{g} / 2 k T} T^{-3 / 2}\right. \\
& \left.+(3 / 2) \cdot C B e^{-E_{g} / 2 k T} T^{1 / 2}\right]>0
\end{aligned}
$$

Таким образом, если для пленки и монокристалла теллура при приведенных выше выражениях для $n$ и $\mu$ $d \sigma / d T>0$ в исследуемом интервале температур, то для НК Те в общем случае выражения для $n(T)$ и $\mu(T)$ должны быть иными в интервале температур, в котором выполняется условие $d \sigma / d T<0$. Очевидно, что зависимость $n(T)$ останется прежней, а выражение для $\mu(T)$, несомненно, подлежит пересмотру.

Известно, что для неограниченной среды, когда рассеяние на границах образца можно не учитывать, подвижность носителей является характеристикой вещества, не зависит от формы и размеров образца и равна $\mu=(e / 2 m)(\bar{l} / \bar{v})$, где $\bar{l}-$ средняя длина свободного пробега, $\bar{v}$ - средняя скорость теплового движения носителей заряда. Однако положение может существенно измениться, если длина свободного пробега, определяемая процессами рассеяния внутри объема (на фононах, дефектах, примесях), становится сравнимой с поперечными размерами образца или их превосходит. В этом случае рассеяние носителей на границах образца будет ограничивать длину свободного пробега. В связи с этим можно полагать, что длина свободного пробега носителей в низкоразмерных структурах, к которым относятся и НК, значительно уменьшится и физика рассеяния носителей заряда будет существенно отличаться по сравнению с трехмерным случаем.

Сопротивление низкоразмерных образцов $(R)$ состоит из объемного сопротивления $\left(R_{O}\right)$, определяемого процессами рассеяния в объеме образца, и добавочного сопротивления $\left(R_{d}\right)$ [22,23]: $R=R_{O}+R_{d}$. Согласно размерной теории Фукса $[24,25]$ для одномерного низкоразмерного образца, т. е. для НК, имеет место следующее соотношение:

$$
R_{d}=\frac{4}{3} \frac{R_{O} \bar{l}}{d}(1-P), \quad \bar{l} \ll d,
$$

где $\bar{l}$ - длина свободного пробега носителей в объемном образце, $d$ - диаметр НК, $P$ - параметр Фукса $(0 \leq P \leq 1)$, характеризующий усредненную отражательную способность поверхности образца. Параметр $P$ зависит от качества поверхности НК. При $P=1$ отражение электронов от поверхности образца происходит полностью зеркально, а при $P=0-$ полностью диффузно. Кроме того, данный параметр зависит от температуры: $P$ уменьшается от максимального значения при низких температурах до нулевого значения при высоких температурах. При этом параметр диффузности поверхности $Q=1-P$ растет от $Q=1-P$ до $Q=1$. Температурная зависимость параметра $P$ обусловлена тепловой „шероховатостью“ поверхности НК, приобретаемой вследствие появления поверхностных фононов и усиливающейся с ростом температуры [25].

Высококачественную поверхность НК можно искусственно „испортить“ травлением, что в свою очередь приведет к повышению сопротивления НК, свидетельствуя об увеличении диффузности поверхности. Диффузность поверхности может быть задана и механизмом роста НК в процессе их выращивания. На рис. 5 представлено полученное в растровом электронном микроскопе (РЭМ) изображение двух боковых граней НК Те с общим ребром. Из рисунка видно, какую развитую („диффузную“) поверхность имеет НК Те в нашем случае. Отметим, что в оптическом микроскопе боковые граны НК Те просматриваются как зеркально-гладкие.

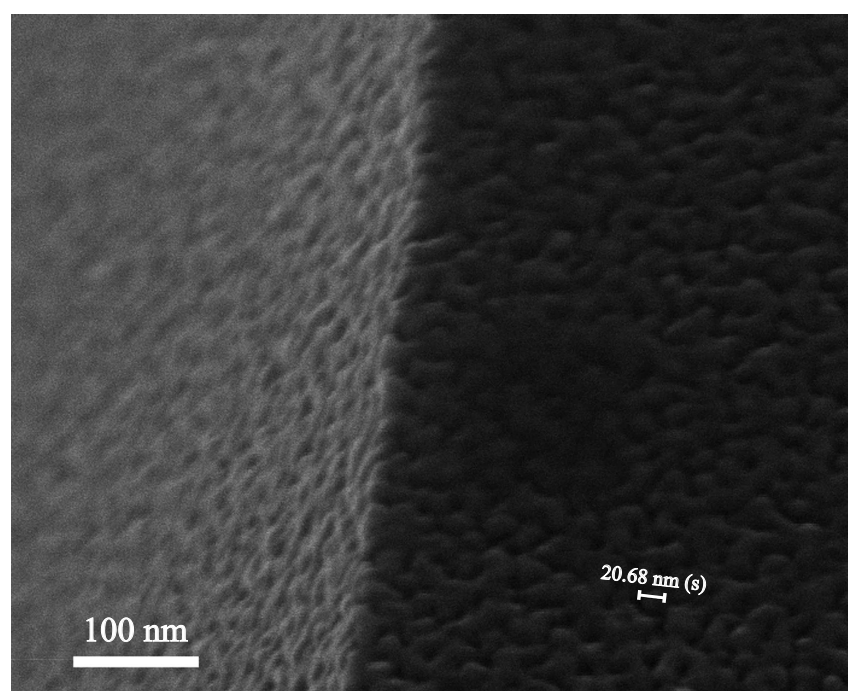

Рис. 5. Изображение в растровом электронном микроскопе боковой поверхности НК Те. 


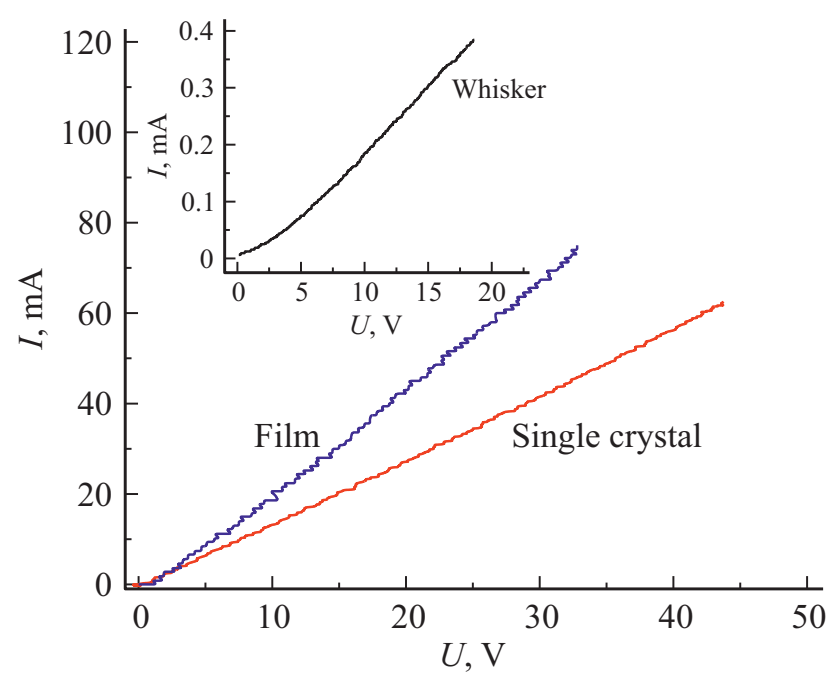

Рис. 6. Вольт-амперные характеристики образцов теллура.

Таким образом, для НК Те добавочное сопротивление $R_{d}$ играет сравнимую с $R_{O}$ роль в температурной зависимости проводимости, т.е. добавочное сопротивление $R_{d}=\left(4 R_{O} \bar{l} / 3 d\right) Q$ с учетом того, что $Q$ растет с температурой, становится определяющим ход температурной зависимости в НК Те (рис. 4). Рост электропроводности НК Те в области $236-273 \mathrm{~K}$ объясняется превалированием составляющей проводимости, обусловленной экспоненциальным ростом концентрации собственных носителей. Наблюдаемый в интервале температур 236-273 K менее крутой ход температурной зависимости НК Те по сравнению с кривыми в случаях пленки и монокристалла, по-видимому, обусловлен продолжающимся ростом степени диффузного рассеяния носителей на боковых гранях НК Те.

Здесь возникает закономерный вопрос - почему при сравнимых значениях толщины эпитаксиальной пленки (13 мкм, см. рис. 2) и диаметра (размера поперечного сечения) НК Те (10 мкм, см. рис. 1) размерный эффект проводимости не проявляется для пленки? Вероятно, это связано с меньшей удельной поверхностью пленки по сравнению с НК.

Рассмотрим общий случай, когда подвижность $\mu$ в выражении для проводимости $\sigma=e n \mu$ НК можно принять как некую среднюю величину от подвижности носителей объемной части образца и подвижности носителей в приповерхностном слое, где формируется добавочное сопротивление $R_{d}$. Представим такую суммарную подвижность в обобщенном виде: $\mu(T)=B T^{-m}, m>0$, тогда

$$
\begin{gathered}
\sigma=e C e^{-E_{g} / 2 k T} B T^{-m}, \\
\frac{d \sigma}{d T}=e\left[\left(C B E_{g} / 2 k T^{2}\right) e^{-E_{g} / 2 k T} T^{-m}\right. \\
\left.-m C B T^{-(m+1)} e^{-E_{g} / 2 k T}\right] \\
=e C B T^{-(m+1)} e^{-E_{g} / 2 k T}\left(E_{g} / 2 k T-m\right) .
\end{gathered}
$$

Численное значение $m$ можно найти из условия минимума температурной зависимости проводимости $(d \sigma / d T)=0$ НК Те при температуре $T_{\min }=236 \mathrm{~K}$ : $m=\left(E_{g} / 2 k T_{\min }\right)$. С учетом значения ширины запрещенной зоны для объемного теллура $\left(E_{g}=0.34 \mathrm{\jmath B}\right)$ вычисления дают $m=8.3$.

Исследовались также динамические вольт-амперные характеристики $I(U)$ образцов теллура приложением к ним импульсов электрического поля треугольной формы длительностью 25-50 мкс в интервале температур 77-273 К. На рис. 6 представлены вольт-амперные характеристики (BAX) образцов Те, измеренные при азотной температуре $(77 \mathrm{~K})$. Все ВАХ образцов в исследованном интервале температур и напряжений линейны.

\section{4. Заключение}

Исследованы температурные зависимости проводимости нитевидного кристалла, эпитаксиальной пленки теллура, полученных термовакуумным напылением в среде водорода, и монокристалла, выращенного методом Чохральского, в интервале температур 77-273 K. Ход температурной зависимости проводимости НК Те коренным образом отличается от кривых для пленки и монокристалла. Электропроводность монокристалла и пленки монотонно растет от 77 до $200 \mathrm{~K}$, далее до температуры $273 \mathrm{~K}$ наблюдается резкий рост электропроводности, связанный с термическим возбуждением собственных носителей заряда. Электропроводность НК Те падает почти линейно от 77 до $236 \mathrm{~K}$, затем до температуры $273 \mathrm{~K}$ следует более плавный рост по сравнению с ростом электропроводности пленки и монокристалла Те в этом же интервале температур. Спад электропроводности НК Те в указанном интервале температур обусловлен проявлением классического размерного эффекта: возникает добавочное сопротивление, связанное с диффузным рассеянием носителей на боковых гранях НК, усиливающимся с ростом температуры. Шероховатость граней НК Те подтверждается исследованием морфологии.

Возможность изменения в широких пределах технологических параметров в предложенном нами методе синтеза микроструктур теллура также позволяет получать полые нитевидные кристаллы (микротрубки) теллура диаметром 10-100 мкм, длиной $>1$ см и с толщиной стенки $\sim 1$ мкм. Данные образцы характеризуются еще большей удельной поверхностью по сравнению со сплошными НК Те, благодаря чему размерные эффекты в них будут выражены сильнее. Получение, изучение свойств и практическое применение данных структур теллура представляется перспективным направлением исследований.

\section{Финансирование работы}

Работа выполнена при поддержке проекта РФФИ № 18-02-00808A. 


\section{Конфликт интересов}

Авторы заявляют, что у них нет конфликта интересов.

\section{Список литературы}

[1] P. Grosse. Die Festkörper Eigenschaften von Tellur. Springer Tracts in Modern Physics (Berlin, 1969).

[2] I.Shih, C.H. Champness. J. Cryst. Growth, 44 (4), 492 (1978).

[3] Р.В. Парфеньев, И.И.Фарбштейн, И.Л. Шульпина. ФТТ, 44 (7), 1190 (2002).

[4] M.J. Capers, M. White. Thin Sol. Films, 8, 353 (1971).

[5] T. Hristova-Vasileva, I. Bineva, R. Todorov, A. Dinescu, C. Romanitan. 20th Intern. School on Condensed Matter Physics [J. Phys.: Conf. Ser. (Россия, 2019) p. 1186].

[6] М.С. Бреслер, В.Г. Веселаго, Ю.В. Косичкин, Г.Е. Пикус, И.И. Фарбштейн, С.С. Шалыт. ЖЭТФ, 57 (5), 1479 (1969).

[7] S. Tutihasi, S.G. Roberts, R.S. Keeres, R.E. Drens. Phys. Rev., 177 (3), 1143 (1969).

[8] R. Viswanathan, R. Balasubramanian, D. Darwin Albert Raj, M. Sai Baba, T.S. Lakshmi Narasimhan. J. Alloys Compd., 603, 75 (2014).

[9] H. Zhen, Y.Yuan, L. Jian-Wei, Y. Shu-Hong. Chem. Soc. Rev., 46, 2732 (2017).

[10] K.R. Sapkota, P. Lu, L.D. Medlin, G.T. Wang. APL Mater., 7, 081103 (2019)

[11] P. Yuanyuan, G. Shiyuan, Li Yang, Lu Jing. Phys. Rev., 98, 085135 (2018)

[12] Z. Zhili, C. Xiaolin, Y. Seho, C. Jinglei, D. Yawei, N. Chunyao, G. Zhengxiao, X. Maohai, L. Feng, C. Jun-Hyung, Yu Jia, Z. Zhenyu. Phys. Rev. Lett., 119, 106101 (2017).

[13] Wu Wenzhuo, Q. Gang, W. Yixiu, W. Ruoxing, Y. Peide. Chem. Soc. Rev., 47, 7203 (2018).

[14] W. Dawei, Y. Aijun, L. Tiansong, F. Chengyu, P. Jianbin, Liu Zhu, Chu Jifeng, Y. Huan, W. Xiaohua, R. Mingzhe, K. Nikhil. J. Mater. Chem. A, 10, 1039 (2019).

[15] S. Chenfei, L. Yihang, W. Jiangbin, X. Chi, C. Dingzhou, L. Zhen, L. Qingzhou, L. Yuanrui, W. Yixiu, C. Xuan, K. Hiroyuki, Sh. Fuyuki, K. Aravind, K. Rajiv, N. Aiichiro, D. Priya, R.A. Vashishta, Mor, N. Ahmad, H.W. Abbas, W. Wenzhuo, Z. Chongwu. ACS Nano, 14, 303 (2020).

[16] А.М. Исмаилов, И.М. Шапиев, М.Х. Рабаданов, И.Ш. Алиев. Письма ЖТФ, 41 (2), 64 (2015).

[17] W. Xiao Ping, L. Yuan, Sh. Min Zhou, Sh. Yun Lou, Y. Qiang Wang, T. Gao, Y. Biao Liu, X. Jing Shi. Nanopart. Res., 14, 1009 (2012).

[18] T. Il Lee, S. Lee, E. Lee, S. Sohn, Y. Lee, S. Lee, G. Moon, D. Kim, Y. Sang Kim, J. Min Myoung, Z. Lin Wang. Adv. Mater., 25, 2920 (2013).

[19] Р.В. Даттон, Р.С. Мюллер. В сб.: Технология толстых $и$ тонких пленок (М., Мир, 1972) с. 162.

[20] Р.В. Парфеньев, Когарский, И.И. Фарбштейн, С.С. Шалыт. ФTT, 3 (8), 2501 (1961)

[21] N. Chaudhuri. Indian J. Pure Appl. Phys., 3, 50 (1965).

[22] Дж. Займан. Электроны и фбононы (М., ИЛ, 1962).

[23] Ф.Ф. Грин. Поверхностные свойства твердых тел (М., Мир, 1972).

[24] K. Fucks. Proc. Cambr. Phil. Soc., 34, 100 (1938).

[25] Ю.П. Гайдуков. УФН, 142 (4), 571 (1984).

\section{Temperature dependence of conductivity of tellurium whiskers}

\author{
M.R. Rabadanov ${ }^{1}$, A.A. Stepurenko ${ }^{2}$, \\ A.E. Gummetov ${ }^{2}$, A.M. Ismailov ${ }^{1}$ \\ ${ }^{1}$ Dagestan State University, \\ 367003 Makhachkala, Russia \\ ${ }^{2}$ Institute of Physics, Dagestan Federal Research \\ Centre, \\ Russian Academy of Sciences, \\ 367003 Makhachkala, Russia
}

Abstract In the temperature range $77-273 \mathrm{~K}$, a comparative analysis of the electrical conductivity of a whisker, an epitaxial film, and a single crystal of tellurium was undertaken. The electrical conductivity of the film and the single crystal increased monotonically up to $200 \mathrm{~K}$, then began to rise steeply, corresponding to thermal excitation of intrinsic carriers. The electrical conductivity of whiskers decreased with increasing temperature to $230 \mathrm{~K}$, after which it began to increase more gradually. It is assumed that in the case of tellurium whiskers, the classical size effect took place: the decrease in electrical conductivity was due to diffuse scattering of carriers by the lateral surface of the tellurium crystal and was intensified with increasing temperature. The uneven, tightly-convoluted surface of the samples was shown in images produced in a scanning electron microscope in the nanometer range.

Редактор Л.В. Шаронова 